\title{
Endo-permutation modules as sources of simple modules
}

\author{
Nadia Mazza ${ }^{1}$ \\ Institut de Mathématiques \\ Université de Lausanne \\ CH-1015 Lausanne \\ e-mail : Nadia.Mazza@ima.unil.ch
}

August 26, 2005

\footnotetext{
${ }^{1}$ This work is a part of a doctoral thesis in preparation at the University of Lausanne, under the supervision of Prof. Jacques Thévenaz
} 


\begin{abstract}
The source of a simple $k G$-module, for a finite $p$-solvable group $G$ and an algebraically closed field $k$ of prime characteristic $p$, is an endo-permutation module (see [Pu1] or [Th]). L. Puig has proved, more precisely, that this source must be isomorphic to the cap of an endo-permutation module of the form $\bigotimes_{Q / R \in \mathcal{S}} \operatorname{Ten}_{Q}^{P} \operatorname{Inf}_{Q / R}^{Q}\left(M_{Q / R}\right)$, where $M_{Q / R}$ is an indecomposable torsion endo-trivial module with vertex $Q / R$, and $\mathcal{S}$ is a set of cyclic, quaternion and semi-dihedral sections of the vertex of the simple $k G$-module. At present, it is conjectured that, if the source of a simple module is an endo-permutation module, then it should have this shape. In this paper, we are going to give a method that allow us to realize explicitly the cap of any such indecomposable module as the source of a simple module for a finite $p$-nilpotent group.
\end{abstract}




\section{Introduction}

1978, E. Dade introduced the concept of endo-permutation module, in the field of modular representation theory (see [Da1]). The study of these modules occupy at present an important position in representation theory, as they seem to appear very often. In particular, they appear as sources of simple modules for $p$-solvable groups, as it has been proved in [Pu1] and section 30 in [Th]. This fact raises then the following question: is any indecomposable endo-permutation module the source of a simple module, for some finite group? In other words, given a prime $p$, a finite $p$-group $P, k$ an algebraically closed field of characteristic $p$ and an indecomposable endo-permutation module $M$ with vertex $P$, does there exist a finite group $G$ having $P$ as $p$-Sylow subgroup and a simple $k G$-module $L$, such that $M$ is isomorphic to a direct summand of the restriction of $L$ to $P$ ? We can't answer this question at the moment. However, if we consider a large subfamily of torsion endo-permutation modules, then we are able to realize explicitly these modules as sources, as we will prove later. Let us also point out that, in order to realize explicitly such situations, we took some inspiration from Dade's methods, used in [Da2].

Let us fix the notations and recall some basic facts we are going to use. Let $p$ be a prime, $\mathcal{O}$ a complete discrete valuation ring, with quotient field $K$ of characteristic 0 and residue field $k$, algebraically closed, of characteristic $p$ ( $K$ is supposed to be "big enough" for the considered groups). If $G$ is a finite group, $H$ a subgroup of $G, M$ a $k G$-module and $N$ a $k H$-module, then we write $M \downarrow_{H}^{G}$ the restriction of $M$ from $k G$ to $k H, N \uparrow_{H}^{G}$ the induction of $N$ from $k H$ to $k G$ and $\operatorname{Ten}_{H}^{G} N$ the tensor induction of $N$ from $k H$ to $k G$. Moreover, if $H \triangleleft G$ and $L$ is a $k[G / H]$-module, then we denote by $\operatorname{Inf}_{G / H}^{G} L$ the inflation of $L$ from $k[G / H]$ to $k G$. Recall that if $g \in G$, then ${ }^{g} N$, also written $g \otimes N$, is the conjugate module of $N$ by $g$ and has a structure of $k\left[{ }^{g} H\right]$-module given by ${ }^{g} h \cdot g_{n}=g(h \cdot n)$, for all $h \in H$ and $n \in N$, where $\cdot \operatorname{denotes}$ the action of $H$ on $N$ and ${ }^{g} h=g h g^{-1}$. If $M_{K}$ and $M_{K}^{\prime}$ are two $K G$-modules, then we have a scalar product (over $K$ ) defined by

$$
\left(M_{K}, M_{K}^{\prime}\right)_{G}=\sum_{g \in G} \operatorname{Tr}\left(g, M_{K}\right) \operatorname{Tr}\left(g^{-1}, M_{K}^{\prime}\right),
$$

where $\operatorname{Tr}\left(g, M_{K}\right)$ is the trace of the $K$-linear automorphism corresponding to the action of $g$ on $M_{K}$. Note that we consider only finitely generated modules. In case $M_{K}$ and $M_{K}^{\prime}$ are simple $K G$-modules, then the orthogonality relations give $M_{K} \cong M_{K}^{\prime} \Longleftrightarrow\left(M_{K}, M_{K}^{\prime}\right)_{G}=1$ (else $\left.\left(M_{K}, M_{K}^{\prime}\right)_{G}=0\right)$. We admit that the reader is familiar with elementary notions of representation theory of finite groups and we come now to endo-permutation modules. A $k P$-module $M$ is endo-permutation if the $P$-algebra $\operatorname{End}_{k} M$ is a permutation $k P$-module (i.e. there is a $P$-invariant $k$-basis). Moreover, if $M$ is indecomposable, we say that it has vertex $P$ if $M$ is not projective relative to any proper subgroup of $P$. If $M$ is an endo-permutation $k P$-module with at least one indecomposable direct summand $M_{0}$ with vertex $P$, then this summand is unique, up to isomorphism and it is called the cap of $M$. This allows us to define an equivalence relation on theses modules:

$$
M \sim N \Longleftrightarrow M_{0} \cong N_{0}
$$

We write $D(P)$ the abelian group formed by the equivalence classes of endo-permutation $k P$ modules having an indecomposable direct summand of vertex $P$ and we call it the Dade group of $P$. The composition law is given by $[M]+[N]=[M \otimes N]$, the neutral element is the class of the trivial $k P$-module $k$ (containing also all permutation $k P$-modules having a trivial direct 
summand) and $-[M]=\left[M^{*}\right]$, where $M^{*}=\operatorname{Hom}_{k}(M, k)$ is the dual of $M$. We denote by $D^{t}(P)$ the torsion subgroup of $D(P)$ and by $T(P)$ the subgroup of $D(P)$ consisting of equivalence classes of endo-trivial $k P$-modules, i.e. $k P$-modules $M$ such that $\operatorname{End}_{k} M \cong k \oplus L$, for a free $k P$-module $L$ (notice that equivalence classes of endo-trivial modules are smaller than the corresponding classes in $D(P))$. For instance, the syzygies $\Omega_{P}^{n}(k), n \in \mathbb{Z}$ of the trivial module are indecomposable endotrivial $k P$-modules with vertex $P$. The structure of $D(P)$ is strongly related to the structure of $T(P)$, as it can be noticed in the following theorem, due to Dade (see [Da1]), which classifies all endo-permutation modules in the case of an abelian $p$-group.

Theorem 1.1 [Dade, [Da]] Let $P$ be an abelian p-group.

1. $T(P)$ is generated by $\left[\Omega_{P}^{1}(k)\right]$. Thus $T(P)$ is trivial, if $|P|=2$, cyclic of order 2 , if $P$ is cyclic of order $\geq 3$, and infinite cyclic otherwise.

2. $D(P)$ is isomorphic to the direct sum of the groups $T(P / Q)$, where $Q$ runs over the set of proper subgroups of $P$.

Now that we have recalled all the notions we are going to use, we can explain how we are going to proceed to realize endo-permutation modules as sources. We start by constructing some examples of finite $p$-nilpotent groups $G$ having a given $p$-group $P$ as $p$-Sylow subgroup. Then we build, for each group $G$, a simple $k G$-module with vertex $P$ and determine its source, that we know to be endo-permutation. Then we prove that, in a sense that will become clear later, the operations of inflation, tensor induction and tensor product provide us a kind of "tools" that allow us to realize explicitly many other situations from known ones. Finally we note that the previous examples, combined with these "tools", lead us directly to the main result:

Theorem Let $p$ be a prime, $P$ a finite $p$-group and $k$ an algebraically closed field of characteristic $p$. Then any indecomposable endo-permutation $k P$-module $M$ with vertex $P$ that is a direct summand of a $k P$-module of the form $\bigotimes_{Q / R \in \mathcal{S}} \operatorname{Ten}_{Q}^{P} \operatorname{Inf}_{Q / R}^{Q}\left(M_{Q / R}\right)$, where $M_{Q / R}$ is an indecomposable torsion endo-trivial module with vertex $Q / R$, and $\mathcal{S}$ is a set of sections of $P$ that are cyclic, or also quaternion or semi-dihedral, in case $p=2$, can be explicitly realized as source of a simple module with vertex $P$ for some finite $p$-nilpotent group.

To conclude this introductive section, we would try to justify our choice for concentrating on torsion endo-permutation modules. At present, it is conjectured that, if the source of a simple module is an endo-permutation module, then its class should be a torsion element in the Dade group. This result has already been proved for finite $p$-solvable groups (cf. [Pu1]). Moreover, it is also conjectured that there are no other indecomposable torsion endo-permutation modules as the ones that are isomorphic to the cap of a module of the form $\bigotimes_{Q / R \in \mathcal{S}} \operatorname{Ten}_{Q}^{P} \operatorname{Inf}_{Q / R}^{Q}\left(M_{Q / R}\right)$, as in our main theorem. Hence, if both conjectures hold, then we would have proved that any endo-permutation module that can be the source of a simple module, namely any torsion endopermutation module, can be explicitly realized as the source of a simple module for a finite $p$ nilpotent group. Let us finally point out the fact that, in a talk given at the LMS Durham Symposium on representations of finite groups and related algebras (01-11 July 2002), Jon Carlson proved a result on $T(P)$ which implies that the second conjecture holds for $p$ odd. 


\section{Construction of finite $p$-nilpotent groups}

Let $p$ be a prime number and $P$ a cyclic, quaternion or semi-dihedral $p$-group. Following Dade's ideas, exposed in [Da2], we are going to build a finite $p$-nilpotent group $G=Q \rtimes P$, with $P$ as $p$-Sylow subgroup and $Q$ an extra-special $q$-group of exponent $q$ and order $q^{3}$, if $P$ is cyclic or quaternion, and $q^{5}$, if $P$ is semi-dihedral, for a "well chosen" prime number $q$.

Let us recall some useful properties about automorphisms of extra-special $q$-groups of order $q^{3}$ and exponent $q$, for an odd prime $q$. Let $Q$ be generated by two elements $x$ and $y$ of order $q$, and set $z=[y, x]$, where $[y, x]=y x y^{-1} x^{-1}$. Then $z$ has order $q$ and generates the center of $Q$, which coincides with the subgroup $Q^{\prime}$ generated by the commutators. The quotient $Q / Q^{\prime}$ is a symplectic $\mathbb{F}_{q}$-vector space of dimension 2 , for the symplectic form [, ] induced by taking the commutator of two elements. In the basis $\{\bar{x}, \bar{y}\}$ of $Q / Q^{\prime}$ (if $g \in Q$, we write $\bar{g}$ its class in $Q / Q^{\prime}$ ), [ , ] corresponds to the matrix $\left(\begin{array}{cc}0 & 1 \\ -1 & 0\end{array}\right)$. Then, the group $\operatorname{Aut}\left(Q / Q^{\prime}\right)$ of automorphisms of $Q / Q^{\prime}$ is the symplectic group $\operatorname{Sp}_{2}\left(\mathbb{F}_{q}\right)$, which coincides with $\mathrm{SL}_{2}\left(\mathbb{F}_{q}\right)$. Let $\operatorname{Aut}_{Q^{\prime}}(Q)$ be the subgroup of $\operatorname{Aut}(Q)$ formed by the automorphisms of $Q$ which are the identity on restriction to $Q^{\prime}$. We have a group homomorphism

$$
\pi: \operatorname{Aut}_{Q^{\prime}}(Q) \longrightarrow \mathrm{SL}_{2}\left(\mathbb{F}_{q}\right), \pi(\varphi)(\bar{g})=\overline{\varphi(\tilde{g})}, \forall \tilde{g} \in \bar{g} \in Q / Q^{\prime} \text { and } \forall \varphi \in \operatorname{Aut}_{Q^{\prime}}(Q) .
$$

Moreover, $\pi$ is surjective. Indeed, let us consider

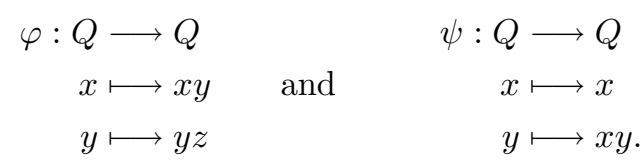

It is easy to check that $\varphi$ and $\psi$ belong to $\operatorname{Aut}_{Q^{\prime}}(Q)$ and, since $\mathrm{SL}_{2}\left(\mathbb{F}_{q}\right)$ is generated by

$$
e=\left(\begin{array}{ll}
1 & 0 \\
1 & 1
\end{array}\right) \quad \text { and } \quad f=\left(\begin{array}{ll}
1 & 1 \\
0 & 1
\end{array}\right)
$$

(see Theorem 2.8.4 in [Go]), the surjectivity of $\pi$ is immediate.

We also notice that $\operatorname{Ker}(\pi)$ is isomorphic to $Q / Q^{\prime}$. Indeed, the two following maps are injective group homomorphisms:

$$
\begin{aligned}
\operatorname{conj}: Q / Q^{\prime} & \longrightarrow \operatorname{Ker}(\pi) \\
\bar{g} & \longmapsto \operatorname{conj}(\bar{g})
\end{aligned} \quad \text { and } \quad \begin{aligned}
\tau: \operatorname{Ker}(\pi) & \longrightarrow \operatorname{Hom}_{\mathbb{F}_{q}}\left(Q / Q^{\prime}, Q^{\prime}\right) \\
\varphi & \longmapsto \tau(\varphi),
\end{aligned}
$$

where $\operatorname{conj}(\bar{g})(h)=\tilde{g}_{h}=\tilde{g} h \tilde{g}^{-1}$ and $\tau(\varphi)(\bar{g})=\tilde{g}^{-1} \varphi(\tilde{g})$, for a representative $\tilde{g} \in \bar{g}, \forall \bar{g} \in$ $Q / Q^{\prime}, \forall h \in Q$ and $\forall \varphi \in \operatorname{Ker}(\pi)$. As $\mathbb{F}_{q}$-vector space, $Q^{\prime}$ is isomorphic to $\mathbb{F}_{q}$. This implies that $\operatorname{Hom}_{\mathbb{F}_{q}}\left(Q / Q^{\prime}, Q^{\prime}\right)$ is isomorphic to the dual of $Q / Q^{\prime}$ and thus to $Q / Q^{\prime}$ as $\mathbb{F}_{q^{-}}$-vector space. In particular, it follows that $|\operatorname{Ker}(\pi)| \leq\left|Q / Q^{\prime}\right|$ and so conj and $\tau$ are isomorphisms. We get then a short exact sequence:

$$
0 \longrightarrow Q / Q^{\prime} \longrightarrow \operatorname{Aut}_{Q^{\prime}}(Q) \longrightarrow \mathrm{SL}_{2}\left(\mathbb{F}_{q}\right) \longrightarrow 0
$$

Moreover, if $p$ and $q$ are distinct prime numbers and $P$ is a $p$-subgroup of $\mathrm{SL}_{2}\left(\mathbb{F}_{q}\right)$, then we get another short exact sequence by restriction:

$$
(*) \quad 0 \longrightarrow Q / Q^{\prime} \longrightarrow \pi^{-1}(P) \longrightarrow P \longrightarrow 0 .
$$


It follows from the Schur-Zassenhaus Theorem (see Corollary 8.40 in $[\mathrm{CR}]$ ) that this sequence splits, because $Q / Q^{\prime}$ and $P$ have coprime orders and $Q / Q^{\prime}$ is abelian. So we have an injective map $\sigma: P \longrightarrow \operatorname{Aut}_{Q^{\prime}}(Q)$, such that $\pi \sigma=\operatorname{Id}_{P}$.

Recall that $\mathrm{SL}_{2}\left(\mathbb{F}_{q}\right)$ has order $q\left(q^{2}-1\right)$ and its 2-Sylow subgroups are generalized quaternion. Thus the 2-subgroups of $\mathrm{SL}_{2}\left(\mathbb{F}_{q}\right)$ are either generalized quaternion or cyclic. From now on, we will drop the word "generalized" in the expression "generalized quaternion group".

Lemma 2.1 Let $p$ and $q$ be two distinct prime numbers, $P$ be a finite cyclic $p$-group or, if $p=2$, cyclic or quaternion, such that $P$ is isomorphic to a subgroup of $\mathrm{SL}_{2}\left(\mathbb{F}_{q}\right)$, and let $Q$ be an extraspecial q-group of order $q^{3}$ and exponent $q$. Then there exists an injective group homomorphism $\sigma: P \longrightarrow \operatorname{Aut}(Q)$. Moreover, we can choose $\sigma$ such that the restriction of $\sigma(u)$ to $Q^{\prime}$ is the identity morphism, for all $u \in P$.

Following Dade's ideas (cf. [Da2]), if we choose a prime number $q$ such that $q \equiv-1(\bmod |P|)$, then, by the above Lemma, $P$ identifies with a subgroup of $\operatorname{Aut}(Q)$, whose elements fix $Q^{\prime}$. This defines a semi-direct product $G=Q \rtimes P$, where $P$ is a $p$-Sylow subgroup, acting by conjugation on the normal $q$-subgroup $Q$ and $Q^{\prime}$ centralizes $P$. We can also notice, in case $p=2$ and $P$ is quaternion, that we can also consider a 2-nilpotent group, with the same features, if we take a prime number $q$ with $q \equiv \frac{|P|}{2}+1(\bmod |P|)$ (then $P$ identifies with a 2-Sylow subgroup of $\operatorname{SL}_{2}\left(\mathbb{F}_{q}\right)$ ). The choices of congruences for the prime $q$ will be motivated in section 4 . Let us state some properties of the $p$-nilpotent groups $G=Q \rtimes P$.

Lemma 2.2 With the same notation as above, the group $G$ satisfies the following properties.

a) We can choose a section $\sigma: P \longrightarrow \pi^{-1}(P)$ of $(*)$ such that $\sigma(w)$ has order $p$ and no fixed point on $Q \backslash Q^{\prime}$, for a generator $w$ of the unique subgroup of $P$ of order $p$. In other words, $w$ and $g \in Q$ commute if and only if $g \in Q^{\prime}$.

b) The normalizer $N$ of $P$ in $G$ is equal to $Q^{\prime} \times P$.

c) $P$ is a trivial intersection subgroup of $G$.

Proof. a) Assume that $P$ is cyclic of order $p^{n}$, generated by $u$ and write $w=u^{p^{n-1}}$. As $\sigma$ is injective, $\sigma(u)$ has order $p^{n}$, and so $\sigma(w)$ has order $p$.

If $\sigma(w)$ has a fixed point on $Q \backslash Q^{\prime}$, then $\pi(\sigma(w)) \in \mathrm{SL}_{2}\left(\mathbb{F}_{q}\right)$ has an eigenvector for the eigenvalue 1 and so is conjugate to a matrix of order $p$ of the form $A=\left(\begin{array}{ll}1 & a \\ 0 & 1\end{array}\right)$, for an $a \in \mathbb{F}_{q}$. But then, we have $A^{p}=\mathrm{Id} \Longleftrightarrow p a=0 \Longleftrightarrow a=0$, since $p$ is invertible in $\mathbb{F}_{q}$. It follows that $A=\mathrm{Id}$, which contradicts the fact that $A$ has order $p$. Thus $\sigma(w)$ has no fixed point on $Q \backslash Q^{\prime}$.

Now, if $p=2$ and $P$ is a quaternion 2-group, then, as before, we can choose $\sigma$ such that $\sigma(w)$ has order 2, i.e. $\sigma(w)=-\mathrm{Id}$. So 1 is not an eigenvalue of $\pi(\sigma(w))$, i.e. $\sigma(w)$ has no fixed point on $Q \backslash Q^{\prime}$.

b) As $G$ is $p$-nilpotent, we have $N=C_{G}(P) \times P$ and so we just need to verify that $C_{G}(P)=Q^{\prime}$. By construction, $Q^{\prime}$ centralizes $P$, so we have $C_{G}(P) \geq Q^{\prime}$. Conversely, if $g \in C_{G}(P)$, then, in particular, $g$ and $w$ commute. By a), it implies that $g \in Q^{\prime}$.

c) Let $g \in Q$ and $s \in{ }^{g} P \cap P$. There exists $s^{\prime} \in P$ such that $s={ }^{g} s^{\prime}$ and so $\left[g, s^{\prime}\right]=1$. Indeed, $\left[g, s^{\prime}\right]=g_{s} s^{\prime}\left(s^{\prime}\right)^{-1}=s\left(s^{\prime}\right)^{-1} \in P$ and $\left[g, s^{\prime}\right]=g\left(s^{\prime} g\right)^{-1} \in Q$ as $Q \triangleleft G$. Thus $\left[g, s^{\prime}\right] \in P \cap Q=\{1\}$. It follows, by a), that $g \in Q^{\prime}$, if $s^{\prime} \neq 1$, i.e. $s \neq 1$, as $w$ is contained in any non trivial subgroup of $P$. 
Now, we are going to construct a finite 2-nilpotent group with a semi-dihedral 2-Sylow subgroup $P=\langle u, v| u^{2^{n-1}}=v^{2}=1, v^{v}=u^{2^{n-2}-1}>$ of order $2^{n}$, with $n \geq 4$. Consider a prime number $q \equiv 2^{n-1}-1\left(\bmod 2^{n}\right)$ and

$$
Q=\left\langle x_{1}, x_{2}, y_{1}, y_{2} \mid x_{i}^{q}=y_{i}^{q}=1,{ }^{y_{i}} x_{i}=x_{i}\left[y_{i}, x_{i}\right],\left[x_{1}, x_{2}\right]=\left[y_{1}, y_{2}\right]=\left[x_{i}, y_{j}\right]=1, \forall 1 \leq i \neq j \leq 2\right\rangle
$$

an extra-special $q$-group of order $q^{5}$, of exponent $q$ and center $Q^{\prime}=<z=\left[y_{1}, x_{1}\right]=\left[y_{2}, x_{2}\right]>$ cyclic of order $q$. We can also consider $Q$ as the central product of two extra-special $q$-groups $Q_{1}$ and $Q_{2}$ of order $q^{3}$, of exponent $q$ and center $Q^{\prime}$, where $Q_{i}$ is the subgroup of $Q$ generated by $x_{i}$ and $y_{i}$, for $i=1$ and 2. There are three maximal subgroups in $P$, one of which is quaternion, call it $R$, generated by $u^{2}$ and $u v$. The previous results allow us to consider a section $\sigma: R \longrightarrow \operatorname{Aut}_{Q_{1}^{\prime}}\left(Q_{1}\right)$ of the split exact sequence $(*)$ above. Notice that we get another injective map $\tilde{\sigma}: R \longrightarrow \operatorname{Aut}_{Q_{2}^{\prime}}\left(Q_{2}\right)$ defined as follows. Let $\theta$ be the isomorphism $Q_{1} \longrightarrow Q_{2}$ mapping $x_{1}$ to $x_{2}$ and $y_{1}$ to $y_{2}$ (and consequently $\theta(z)=z$ ) and let $\lambda$ be the automorphism of $R$, mapping $u^{2}$ to $\left(u^{2}\right)^{-1}$ and $(u v)$ to $\left(u^{2}\right)^{2^{n-3}-1}(u v)$ (it is bijective since $\lambda^{2}=\mathrm{Id}$ ). Then, if

$$
\begin{aligned}
& \sigma: R \longrightarrow \operatorname{Aut}_{Q_{1}^{\prime}}\left(Q_{1}\right), \quad \text { we set } \quad \tilde{\sigma}: R \longrightarrow \operatorname{Aut}_{Q_{2}^{\prime}}\left(Q_{2}\right) \\
& u^{2} \longmapsto \sigma\left(u^{2}\right) \quad u^{2} \longmapsto \theta \sigma\left(\lambda\left(u^{2}\right)\right) \theta^{-1}=\theta \sigma\left(u^{2}\right)^{-1} \theta^{-1} \\
& u v \longmapsto \sigma(u v) \quad u v \longmapsto \theta \sigma(\lambda(u v)) \theta^{-1}=\theta \sigma\left(u^{2}\right)^{2^{n-3}-1} \sigma(u v) \theta^{-1} .
\end{aligned}
$$

Consider then

$$
\begin{aligned}
& P \longrightarrow \operatorname{Aut}_{Q^{\prime}}(Q), \\
& u \longmapsto \sigma_{u} \\
& v \longmapsto \sigma_{v},
\end{aligned}
$$

where $\left.\quad \sigma_{u}\right|_{Q_{1}}=\tilde{\sigma}(u v) \theta,\left.\quad \sigma_{v}\right|_{Q_{1}}=\theta,\left.\quad \sigma_{u}\right|_{Q_{2}}=\sigma(u v) \theta^{-1} \quad$ and $\left.\quad \sigma_{v}\right|_{Q_{2}}=\theta^{-1}$,

and where $\left.\sigma_{g}\right|_{Q_{i}}$ denotes the restriction of $\sigma_{g}$ to $Q_{i}$, for $g \in\{u, v\}$ and $i=1$ and 2 .

This is well defined and extends the maps $\sigma: R \longrightarrow \operatorname{Aut}_{Q_{1}^{\prime}}\left(Q_{1}\right)$ and $\tilde{\sigma}: R \longrightarrow \operatorname{Aut}_{Q_{2}^{\prime}}\left(Q_{2}\right)$. Indeed, we have:

$$
\begin{gathered}
\left.\left(\sigma_{u}^{2}\right)\right|_{Q_{1}}=\left.\left.\left(\sigma_{u}\right)\right|_{Q_{2}}\left(\sigma_{u}\right)\right|_{Q_{1}}=\left(\sigma(u v) \theta^{-1}\right)(\tilde{\sigma}(u v) \theta)= \\
=\sigma(u v) \sigma\left(u^{2}\right)^{2^{n-3}-1} \sigma(u v)=\sigma\left(u^{2}\right)^{2^{n-3}+1} \sigma(u v)^{2}=\sigma\left(u^{2}\right)^{2^{n-3}+1} \sigma\left(u^{2}\right)^{2^{n-3}}=\sigma\left(u^{2}\right)
\end{gathered}
$$

and

$$
\begin{gathered}
\left.\left(\sigma_{u}^{2}\right)\right|_{Q_{2}}=\left.\left.\left(\sigma_{u}\right)\right|_{Q_{1}}\left(\sigma_{u}\right)\right|_{Q_{2}}=\left(\theta \sigma\left(u^{2}\right)^{2^{n-3}-1} \sigma(u v)\right)\left(\sigma(u v) \theta^{-1}\right)= \\
=\theta \sigma\left(u^{2}\right)^{2^{n-3}-1} \sigma(u v)^{2} \theta^{-1}=\theta \sigma\left(u^{2}\right)^{-1} \theta^{-1}=\tilde{\sigma}\left(u^{2}\right) .
\end{gathered}
$$

We also have $\sigma_{v}^{2}=\mathrm{Id}$ and ${ }^{\sigma_{v}} \sigma_{u}=\sigma_{u}^{2^{n-2}-1}$, since $\left.\left(\sigma_{u} \sigma_{v}\right)\right|_{Q_{1}}=\sigma(u v)$ and $\left.\left(\sigma_{u} \sigma_{v}\right)\right|_{Q_{2}}=\tilde{\sigma}(u v)$. It follows that the subgroup of $\operatorname{Aut}_{Q^{\prime}}(Q)$ generated by $\sigma_{u}$ and $\sigma_{v}$ is semi-dihedral of order $2^{n}$. In other words, we get a finite 2-nilpotent group $G$, which is the semi-direct product of $P$ by $Q$.

Moreover, using the same argument as in the proof of the previous Lemma, we have $C_{Q}(P)=Q^{\prime}$ and so $N_{G}(P)=Q^{\prime} \times P$. The next result shows two differences between this construction and the previous one.

Lemma 2.3 $P$ has two conjugacy classes of elements of order 2 . Moreover, $P$ is not a trivial intersection subgroup of $G$. 
Proof. Observe that all non-central elements of $P$ of order 2 can be written as a product $u^{2 a} v$, for an integer $0 \leq a<2^{n-3}$. Now, for any integer $b$, we have

$$
u^{b} v=u^{b}\left({ }^{v} u\right)^{-b} v=u^{2 b\left(1-2^{n-3}\right)} v .
$$

Thus, all non-central elements of order 2 are conjugate in $P$, by a power of $u$, and so $P$ has two conjugacy classes of subgroups of order 2: one containing only the center and the class containing all the others subgroups of order 2 .

For the second statement, consider the conjugacy morphism by the non-central element $v$ of $P$ of order 2, i.e. the map $\sigma_{v}$ described above. We have $\sigma_{v}\left(x_{1} x_{2}\right)=x_{1} x_{2}$, i.e. $v \in{ }^{x_{1} x_{2}} P \cap P$, but $x_{1} x_{2} \notin N_{G}(P)=Q^{\prime} \times P$.

Notice that if ${ }^{g} P \cap P \neq\{1\}$, then ${ }^{g} P \cap P$ is conjugate to $\langle v>$. Indeed, any non-trivial subgroup of $P$ which does not contain $\langle w\rangle$ has order 2 and must then be a conjugate of $\langle v\rangle$. As $\langle w\rangle \leq{ }^{g} P \cap P$, if and only if $g \in N_{G}(P)$, we deduce that ${ }^{g} P \cap P$ is conjugate to $\langle v\rangle$.

Let us summarize the situation. We have build $p$-nilpotent groups of the form $Q \rtimes P$, where $Q$ is an extra-special $q$-group of exponent $q$, for a prime number $q$ satisfying the following data:

- If $P$ is cyclic, then we choose $q \equiv|P|-1(\bmod p|P|)$ and $Q$ of order $q^{3}$.

- If $P$ is quaternion, then we build two finite 2-nilpotent groups by choosing two distinct prime numbers $q$ : first $q \equiv|P|-1(\bmod 2|P|)$, and then $q \equiv \frac{|P|}{2}+1(\bmod |P|)$. In each case, $Q$ has order $q^{3}$.

- If $P$ is semi-dihedral, then we choose $q \equiv \frac{|P|}{2}-1(\bmod |P|)$ and $Q$ of order $q^{5}$.

\section{Construction of simple $k G$-modules}

Let us first recall some basic facts about the simple $k Q$-modules, where $Q$ is an extra-special $q$ group of order $q^{1+2 m}$ and exponent $q$ for a prime number $q$, distinct from $p=\operatorname{char}(k)$, and an integer $m \geq 1$. Write $x_{1}, \ldots, x_{m}, y_{1}, \ldots, y_{m}$ the generators of $Q$ satisfying the relations $x_{i}^{q}=y_{i}^{q}=$ $1,{ }^{y_{i}} x_{i}=x_{i}\left[y_{i}, x_{i}\right],\left[y_{i}, x_{i}\right]=\left[y_{j}, x_{j}\right]$ and $\left[x_{i}, x_{j}\right]=\left[x_{i}, y_{j}\right]=\left[y_{i}, y_{j}\right]=1, \forall 1 \leq i \neq j \leq m$. Let also $z=\left[y_{1}, x_{1}\right]$ be the generator of the center $Q^{\prime}$ of $Q$ (which coincides with the subgroup generated by the commutators) and let $A$ be the elementary abelian normal subgroup of $Q$ of rank $m+1$, generated by $x_{1}, \ldots, x_{m}$ and $z$. We know, by Proposition $7.7 \mathrm{in}[\mathrm{Hu}]$, that $Q$ has $q-1$ simple $k Q$-modules of dimension $q^{m}$ and $q^{2 m}$ simple $k Q$-modules of dimension 1 . Let us describe them. Let $\omega \in k$ be a primitive $q$-th root of unity and consider the degree 1 representation given by:

$$
\begin{aligned}
\psi: A & \longrightarrow k^{*} \\
x_{i} & \longmapsto 1,1 \leq i \leq m \\
z & \longmapsto \omega .
\end{aligned}
$$


We have ${ }^{g} \psi \neq \psi, \forall g \notin A$. In terms of modules, if we write $k_{\psi}$ the $k A$-module of dimension 1 defined by $a \cdot \lambda=\psi(a) \cdot \lambda, \forall a \in A, \forall \lambda \in k$, then this is equivalent to saying that $k_{\psi} ¥{ }^{g} k_{\psi}$. It follows by Clifford theory (see Chapter 11 in [CR]), that the induced $k Q$-module $L=k_{\psi} \uparrow_{A}^{Q}$ is simple, of dimension $q^{m}$. As $k$ is supposed to be algebraically closed, we have $q-1$ distinct primitive $q$-th roots of unity in $k$ and so we get $q-1$ non isomorphic simple $k Q$-modules of dimension $q^{m}$. On the other hand, we get the $q^{2 m}$ simple $k Q$-modules of dimension 1 by considering the representations

$$
\begin{aligned}
\rho: Q & \longrightarrow k^{*} \\
x_{i} & \longmapsto \omega_{i}, \quad 1 \leq i \leq m \\
y_{i} & \longmapsto \theta_{i}, \quad 1 \leq i \leq m \\
z & \longmapsto 1,
\end{aligned}
$$

where $\omega_{i}$ and $\theta_{i}, 1 \leq i \leq m$ are $q$-th roots of unity in $k$.

Before going further, recall that, as $k Q$ is semi-simple, the decomposition map induces a bijection between simple $k Q$ - and $K Q$-modules (see [Se]). In other words, we can lift in characteristic 0 any simple $k Q$-module $L$ into a unique simple $K Q$-module $L_{K}$ (up to isomorphism). It follows that if $M_{K}$ is a simple $K Q$-module which lifts another simple $k Q$-module $M$, then $L \cong M$, if and only if $L_{K} \cong M_{K}$, if and only if the scalar product $\left(L_{K}, M_{K}\right)_{Q}=1$ (otherwise $\left.\left(L_{K}, M_{K}\right)_{Q}=0\right)$. In our situation, $M$ is a simple $k Q$-module of dimension 1 , if and only if $M \downarrow_{Q^{\prime}}^{Q} \cong k$, where $k$ denotes the trivial $k Q$-module. From the above description of the simple $k Q$-modules, we can also notice that any $k Q$-module $M$ of dimension $q^{m}$ is simple, if and only if $M \downarrow_{Q^{\prime}}^{Q} \cong\left(k_{\omega}\right)^{q^{m}}$, where $k_{\omega}$ is the $k Q$-module of dimension 1 defined by $z \cdot \lambda=\omega \lambda, \forall \lambda \in k$, for a primitive $q$-th root of unity $\omega$ (otherwise $M \downarrow_{Q^{\prime}}^{Q} \cong k^{q^{m}}$ ). So we have proved:

Lemma 3.1 Let $L$ and $M$ be two $k Q$-modules of dimension $q^{m}$, lifted to the $K Q$-modules $L_{K}$ and $M_{K}$, respectively. Assume that $L$ is simple. Then, $L$ and $M$ are isomorphic if and only if $\left(L_{K} \downarrow_{Q^{\prime}}^{Q}, M_{K} \downarrow_{Q^{\prime}}^{Q}\right)_{Q^{\prime}}=q^{2 m}$.

The next question that we are going to discuss handles with extension of modules. Indeed, we have constructed simple $k Q$-modules, but we are interested in simple $k G$-modules. In order to get some simple $k G$-modules, we are going to use Theorem IX.4.1 in [Fe], that proves that our simple $k Q$-modules extend uniquely to $k G$. Let us recall this statement.

Theorem 3.2 Let $G=Q \rtimes P$ a finite $p$-nilpotent group, where $Q$ is a $p^{\prime}$-group and $P$ is a $p$-Sylow subgroup of $G$. Let $k$ be a splitting field of $G$ and let $L$ be a simple $P$-invariant $k Q$-module i.e. ${ }^{u} L \cong L$ for all $u \in P$. Then there exists a unique simple $k G$-module $\tilde{L}$, up to isomorphism, such that $\tilde{L} \downarrow_{Q}^{G} \cong L$. Moreover, $\tilde{L} \downarrow_{P}^{G}$ is an endo-permutation module.

Proof. See Theorem IX.4.1 in [Fe].

Let us turn back to the constructed simple $k Q$-module $L$. To prove the existence of a unique extension $\tilde{L}$ of $L$, it suffices to verify that $L$ is $P$-invariant. Let $u \in P$ and consider the $k Q$-modules $L$ and ${ }^{u} L$ of dimension $q^{m}$, with $m=1$, if $P$ is cyclic or quaternion, and $m=2$, if $P$ is semi-dihedral. Recall that ${ }^{u} L$ is a $k Q$-module for the action of $Q$ given by $g \cdot u \otimes l=u \otimes\left(u^{-1} g \cdot l\right), \forall g \in Q, \forall l \in L$. 
Lemma 3.1 tells us that $L \cong{ }^{u} L$ if and only if $\left({ }^{u} L_{K} \downarrow_{Q^{\prime}}^{Q}, L_{K} \downarrow_{Q^{\prime}}^{Q}\right)_{Q^{\prime}}=q^{2 m}$. But $z$ acts on $L$ by the scalar matrix $\omega \mathrm{Id}$, and so does it also on ${ }^{u} L$, as $u$ and $z$ commute. Thus we have

$$
\left({ }^{u} L_{K} \downarrow_{Q^{\prime}}^{Q}, L_{K} \downarrow_{Q^{\prime}}^{Q}\right){ }_{Q^{\prime}}=q^{-1} \sum_{i=0}^{q-1} \operatorname{Tr}\left(z^{i},{ }^{u} L_{K}\right) \operatorname{Tr}\left(z^{-i}, L_{K}\right)=q^{-1} \sum_{i=0}^{q-1}\left(q^{m} \omega^{i}\right)\left(q^{m} \omega^{-i}\right)=q^{2 m} .
$$

We conclude then that $L$ is $P$-invariant and extends to a unique (up to isomorphism) simple $k G$-module $\tilde{L}$ of dimension $q^{m}$. Moreover, as $\operatorname{dim}(\tilde{L})$ and $p$ are coprime, $\tilde{L}$ has vertex $P$.

\section{Source of $\tilde{L}$}

In this section we are going to determine, up to isomorphism, the sources of the simple modules $\tilde{L}$ we constructed in the previous section. We know, by Theorem 30.5 in [Th], that these sources are indecomposable endo-permutation modules. Moreover, Theorem 8.39 and Remark 8.41 in [Pu1] imply that these sources belong to a subset of the set of torsion endo-permutation modules. But, as this latter result is not published, we will not use it in our argumentation. We use the previous notation and set $X \mid Y$, if $X$ and $Y$ are two modules such that $X$ is (isomorphic to) a direct summand of $Y$. We want to find an indecomposable endo-permutation module $S$, with vertex $P$, satisfying $\tilde{L} \mid S \uparrow_{P}^{G}$ and $S \mid \tilde{L} \downarrow_{P}^{G}=\left(\tilde{L} \downarrow_{N}^{G}\right) \downarrow_{P}^{N}$ (by transitivity of restriction), where $N=N_{G}(P)$. It follows, by uniqueness of the cap of an endo-permutation module, that the module $S$ we are looking for must be a direct summand of the restriction to $P$ of the Green correspondent $M$ of $\tilde{L}$, i.e. $M$ is the unique indecomposable direct summand with vertex $P$ of $\tilde{L} \downarrow_{N}^{G}$ such that $\tilde{L} \mid M \uparrow_{N}^{G}$.

The next lemma shows that $M \downarrow_{P}^{N}$ is an indecomposable $k P$-module, and so $M \downarrow_{P}^{N}$ is the source $S$ of $\tilde{L}$.

Lemma 4.1 Let $N=A \times P$ be a finite group where $A$ is an abelian $p^{\prime}$-group and $P$ is a $p$-group, and let $V$ be an indecomposable $k N$-module. Then, $V \downarrow_{P}^{N}$ is indecomposable.

Proof. As $A$ is of order prime to $p$, the group algebra $k A$ is semi-simple. Thus, we can write $V \downarrow_{A}^{N} \cong \bigoplus S_{i}^{n_{i}}$, where $S_{i}^{n_{i}}$ denotes the isotypic component of $V \downarrow_{A}^{N}$ corresponding to the simple $k A$-module $S_{i}$, for all $i$. The group $P$ permutes the isotypic components of $V \downarrow_{A}^{N}$ transitively, since $V$ is indecomposable, and trivially, since $P$ centralizes $A$. Thus $V \downarrow_{A}^{N} \cong S^{n}$ for a simple $k A$-module $S$ and an integer $n \in \mathbb{N}$. Moreover, we have $\operatorname{dim} S=1$ because $A$ is abelian and $S$ simple.

Since $V$ is a $k N$-module and $N=A \times P$, we have a group homomorphism $N \longrightarrow \operatorname{Aut}_{k}\left(S^{n}\right)$ inducing a morphism

$$
P \longrightarrow \operatorname{Aut}_{k A}\left(S^{n}\right) \cong \mathrm{GL}_{n}\left(\operatorname{End}_{k A} S\right) \cong \mathrm{GL}_{n}(k) .
$$

Hence, there exists a $k P$-module $W$ of dimension $n$, satisfying $V \cong S \otimes W$, as $k N$-modules, for the action of $N$ given by $(a u) \cdot(s \otimes w)=(a \cdot s) \otimes(u \cdot w), \forall a \in A, \forall u \in P, \forall s \in S$ and $\forall w \in W$. Then we have $V \downarrow_{P}^{N} \cong W^{\operatorname{dim} S}=W$ and the result follows, because $W$ is indecomposable, since $V \cong S \otimes W$ is. 
Consider the Green correspondent $M$ of $\tilde{L}$ and write

$$
\text { (**) } \quad \tilde{L} \downarrow_{N}^{G} \mid M \uparrow_{N}^{G} \downarrow_{N}^{G} \cong M \oplus(\bigoplus_{g \in[G / N], g \neq 1} \underbrace{g_{M \downarrow_{g_{N}}^{g_{N}} \uparrow^{N}}^{g_{N \cap N}}}_{(I)}) \text {. }
$$

It follows from (**) that $\tilde{L} \downarrow_{N}^{G}$ is isomorphic to the direct sum of $M$ and of indecomposable direct summands of $(I)$.

\subsection{Cyclic case}

Assume that $P$ is cyclic. In the finite $p$-nilpotent group $G$ we constructed, $P$ is a trivial intersection subgroup (see Lemma 2.2). So the direct summands in $(I)$ have trivial vertex, i.e. they are projective $k G$-modules and their dimension is divisible by $|P|$. We then get from (**) the congruence $\operatorname{dim} \tilde{L} \equiv \operatorname{dim} M(\bmod |P|)$. Since $\operatorname{dim} \tilde{L}=q$ and since $M \downarrow_{P}^{N} \cong S$, the choice of $q$ implies that $\operatorname{dim} S \equiv-1(\bmod |P|)$. As $S$ is indecomposable and $\Omega_{P}^{1}(k)$ is the unique indecomposable $k P$-module with dimension congruent to -1 modulo $|P|$, we must have $S \cong \Omega_{P}^{1}(k)$.

\subsection{Quaternion case}

Assume that $P$ is quaternion. In both finite $p$-nilpotent groups having $P$ as 2-Sylow subgroup, $P$ is a trivial intersection subgroup. So, as in the cyclic case, the direct summand in $(I)$ have trivial vertex, i.e. they are projective and their dimension is divisible by $|P|$. If $q \equiv|P|-1(\bmod 2|P|)$, then the relation $(* *)$ implies $\operatorname{dim} S \equiv-1(\bmod |P|)$. If $q \equiv \frac{|P|}{2}+1(\bmod |P|)$, then $(* *)$ implies $\operatorname{dim} S \equiv \frac{|P|}{2}+1(\bmod |P|)$. Let us prove that in both cases $S$ is endo-trivial.

Set $P=<u, v \mid u^{2^{n-1}}=1, u^{2^{n-2}}=v^{2},{ }^{v} u=u^{-1}>\left(\right.$ so $\left.|P|=2^{n}\right)$ and $w=u^{2^{n-2}}$ for the unique element of $P$ of order 2, i.e. the generator of the center $Z$ of $P$. Lemma 2.9 in [CaTh] tells us that it suffices to prove that $S \downarrow_{Z}^{P}$ is endo-trivial and so we just need to know how $w$ acts on $\tilde{L}$. As the calculations are the same for both congruences of $q$, we don't need to distinguish the cases.

Since - Id is the unique involution of $\mathrm{SL}_{2}\left(\mathbb{F}_{q}\right)$, it corresponds to the action of $w$ on $Q$ and so there exist two integers $a$ and $b$ such that $0 \leq a, b<q,{ }^{w} x=x^{-1} z^{a}$ and ${ }^{w} y=y^{-1} z^{b}$. Since $L=k_{\omega} \uparrow_{<x, z>}^{Q}$ for a primitive $q$-th root of unity $\omega$, we can consider the $k$-basis $\left\{y^{i} \otimes 1 \mid 0 \leq i<q\right\}$ of $\tilde{L}$. Set $w \cdot y^{i} \otimes 1=\omega^{(i-l) b}\left(y^{-i-a} \otimes 1\right), \forall 0 \leq i<q$, where $l$ is the unique integer with $0 \leq l<q$ and $2 l \equiv-a(\bmod q)$. This action extends to $Q \rtimes<w>$ by setting $(g w) \cdot y^{i} \otimes 1=g \cdot\left(w \cdot y^{i} \otimes 1\right)$, for all $g \in Q$ and for all $0 \leq i<q$. This gives $\tilde{L}$ the (unique, by Theorem 3.2) structure of $Q \rtimes<w>$-module, as it satisfies:

$$
\begin{gathered}
1 \cdot y^{i} \otimes 1=w^{2} \cdot y^{i} \otimes 1=w \cdot \omega^{(i-l) b}\left(y^{-i-a} \otimes 1\right)=y^{i} \otimes 1, \\
(w x) \cdot y^{i} \otimes 1=\left(x^{-1} z^{a} w\right) \cdot y^{i} \otimes 1=\omega^{(i-l) b-i}\left(y^{-i-a} \otimes 1\right)=w \cdot\left(x \cdot y^{i} \otimes 1\right) \text { and } \\
(w y) \cdot y^{i} \otimes 1=\left(y^{-1} z^{b} w\right) \cdot y^{i} \otimes 1=\omega^{(i+1-l) b}\left(y^{-i-1-a} \otimes 1\right)=w \cdot\left(y \cdot y^{i} \otimes 1\right) .
\end{gathered}
$$


The matrix of the action of $w$ is

$$
(w, \tilde{L})=\left(\begin{array}{rrrrr} 
& \omega^{l b} & & & \\
& \ldots & & 0 & \\
\omega^{-l b} & & & & \omega^{-(l+1) b} \\
& & & \ldots &
\end{array}\right),
$$

with a unique non-zero element, equal to 1 , on the diagonal, in the $(l, l)$ coordinate, i.e. an eigenvector for the eigenvalue 1 . We also notice that the other columns of the matrix pair to form $\frac{q-1}{2} Z$-invariant subspaces $W_{i}=k<y^{i} \otimes 1, y^{-i-a} \otimes 1>$, for all $i$ strictly between $l$ and $\frac{q-1+2 l}{2}$, and on which $Z$ acts freely. As all these subspaces intersect trivially, their sum is direct and form then a $k Z$-submodule of $\tilde{L} \downarrow_{Z}^{G}$. Counting the dimension, we deduce that $\tilde{L} \downarrow_{Z}^{G}$ decomposes as a direct sum $k \oplus(k Z)^{\frac{q-1+2 l}{2}}$ and consequently we have $S \downarrow_{Z}^{P} \cong k \oplus S^{\prime}$, for a free $k Z$-module $S^{\prime}$, by the Krull-Schmidt Theorem. Thus $S$ is endo-trivial.

Let us recall that there are eight indecomposable endo-trivial $k P$-modules with vertex $P$ (up to isomorphism), whose dimensions determine their orders in the Dade group and allow us to recover generators of $T(P)$ (see Section 6 in [CaTh]). Indeed, up to isomorphism, two have dimension $|P|-1$ and order 4: $\Omega_{P}^{1}(k)$ and its dual $\Omega_{P}^{3}(k)$; one has dimension $|P|+1$ and order $2: \Omega_{P}^{2}(k)$ (which is self-dual); two have dimension $\frac{|P|}{2}+1$ and order 2: an "exceptional" indecomposable endotrivial $k P$-module $V$ with vertex $P$ and its dual; two have dimension $\frac{|P|}{2}-1$ and order $2: \Omega_{P}^{1}(V)$ and its dual; and the trivial $k P$-module, of dimension 1 . So, comparing $S$ with the elements in this list we draw the following conclusions. For $q \equiv|P|-1(\bmod 2|P|)$, we have $\operatorname{dim} S=|P|-1$ and $S$ is isomorphic either to $\Omega_{P}^{1}(k)$, or to $\Omega_{P}^{3}(k)$, i.e. $[S]$ has order 4 in $D(P)$. For $q \equiv \frac{|P|}{2}+1(\bmod |P|)$, we have $\operatorname{dim} S=\frac{|P|}{2}+1$ and $S$ is isomorphic either to $V$, or to its dual and $[S]$ has order 2 in $D(P)$. In particular, we notice that the classes of the two modules realized as sources generate all $T(P)$.

\subsection{Semi-dihedral case}

Assume that $P$ is semi-dihedral, say $P=\langle u, v| u^{2^{n-1}}=v^{2}=1, v^{v} u=u^{2^{n-2}-1}>$ of order $2^{n}$, and use the same notation as in section 2 . Recall that $P$ is not a trivial intersection subgroup of $G$, and that the direct summands of $(I)$ have trivial vertex or vertex cyclic of order 2 , conjugate to $<v>$. So, their dimension is divisible by $2^{n-1}$ and the relation $(* *)$ implies that $\operatorname{dim} S \equiv 1\left(\bmod \frac{|P|}{2}\right)$. We want to prove that $S$ is isomorphic to the endo-trivial $k P$-module $\Omega_{P}^{1}\left(\Omega_{P /<v>}^{1}(k)\right)$ of dimension $\frac{|P|}{2}+1$. By injectivity of restriction and results of section 7 in [CaTh], we must show that $\Omega_{R}^{2}(k) \mid S \downarrow_{R}^{P}$ and that $S \downarrow_{E}^{P}$ is endo-trivial, where $R$ is the maximal quaternion subgroup of $P$ and $E$ is an elementary abelian subgroup of $P$ of order 4 (recall that $E$ is unique up to conjugacy).

For $i=1$ and 2 , consider the simple $k Q_{i}$-modules $L_{i}$ of dimension $q$ satisfying $L_{i} \downarrow_{Q^{\prime}}^{Q_{i}} \cong\left(k_{\omega}\right)^{q}$, for the same primitive root $\omega$ appearing in the simple $k Q$-module $L$. We assert that the $k$-vector space $X=L_{1} \otimes L_{2}$ is a $k Q$-module for the action given as follows: $\forall g \in Q, \exists g_{i} \in Q_{i}, i=1,2$ such that $g=g_{1} g_{2}$. We set then $g \cdot l_{1} \otimes l_{2}=g_{1} \cdot l_{1} \otimes g_{2} \cdot l_{2}, \forall l_{1} \otimes l_{2} \in X$. This action is well defined, as $g_{1} g_{2}=h_{1} h_{2}$, if and only if there exists $0 \leq a<q$ such that $g_{1}=h_{1} z^{a}$ and $g_{2}=h_{2} z^{-a}$, and then 
we have $g_{1} g_{2} \cdot l_{1} \otimes l_{2}=h_{1} z^{a} \cdot l_{1} \otimes h_{2} z^{-a} \cdot l_{2}=\omega^{a} \omega^{-a}\left(h_{1} \cdot l_{1} \otimes h_{2} \cdot l_{2}\right)=h_{1} h_{2} \cdot l_{1} \otimes l_{2}$. Moreover, we have $1 \cdot l_{1} \otimes l_{2}=l_{1} \otimes l_{2}$ and $\left(\left(g_{1} g_{2}\right)\left(h_{1} h_{2}\right)\right) \cdot l_{1} \otimes l_{2}=\left(g_{1} g_{2}\right)\left(\left(h_{1} h_{2}\right) \cdot l_{1} \otimes l_{2}\right), \forall g_{i}, h_{i} \in Q_{i}, \forall i=1,2$ and $\forall l_{1} \otimes l_{2} \in X$.

Lemma $4.2 X$ is isomorphic to $L$ and consequently $X$ is a simple $k Q$-module which extends to the $k G$-module $\tilde{L}$.

Proof. By Lemma 3.1, $X \cong L$, if and only if $\left(X_{K} \downarrow_{Q^{\prime}}^{Q}, L_{K} \downarrow_{Q^{\prime}}^{Q}\right)_{Q^{\prime}}=q^{4}$, as $X$ and $L$ have dimension $q^{2}$ and $L$ is simple. We have $z^{i} \cdot\left(l_{1} \otimes l_{2}\right)=\left(z^{i} \cdot l_{1}\right) \otimes l_{2}=\omega^{i}\left(l_{1} \otimes l_{2}\right), \forall l_{1} \otimes l_{2} \in X$ and it follows then that $\left(X_{K} \downarrow_{Q^{\prime}}^{Q}, L_{K} \downarrow_{Q^{\prime}}^{Q}\right)_{Q^{\prime}}=\left(L_{K} \downarrow_{Q^{\prime}}^{Q}, L_{K} \downarrow_{Q^{\prime}}^{Q}\right)_{Q^{\prime}}=q^{4}$.

Let $H=Q \rtimes R$ and $H_{i}=Q_{i} \rtimes R, i=1$ and 2. By construction, $H_{1}$ and $H_{2}$ are isomorphic to the 2-nilpotent group constructed for the quaternion case, with $q \equiv|R|-1(\bmod 2|R|)$.

Lemma 4.3 Let $\tilde{L}_{i}$ be the extension of the simple $k Q_{i}$-module $L_{i}$ to the 2-nilpotent group $H_{i}$, for $i=1$ and 2 . Then $\tilde{L}_{1} \otimes \tilde{L}_{2}$ is the unique simple $k H$-module (up to isomorphism) that extends $X$, for the action of $H$ given by $g_{1} g_{2} s \cdot l_{1} \otimes l_{2}=g_{1} s \cdot l_{1} \otimes g_{2} s \cdot l_{2}, \forall l_{1} \otimes l_{2} \in \tilde{L}_{1} \otimes \tilde{L}_{2}, \forall g_{i} \in Q_{i}, i=1,2$ and $\forall s \in R$. Moreover, $\tilde{L}_{1} \otimes \tilde{L}_{2} \cong \tilde{L} \downarrow_{H}^{G}$.

Proof. By definition of the external tensor product, $\tilde{L}_{1} \otimes \tilde{L}_{2}$ is a $k Q$-module for the action componentwise of $Q$ (see definition 10.15 in $[\mathrm{CR}]$ ) and it is also a $k R$-module for the diagonal action of $R$. So we just need to check that both actions are compatible, i.e. if $s \in R, g_{i} \in Q_{i}, i=1,2$ and $l_{1} \otimes l_{2} \in \tilde{L}_{1} \otimes \tilde{L}_{2}$, then $\left(s g_{1} g_{2}\right) \cdot l_{1} \otimes l_{2}=s \cdot\left(g_{1} g_{2} \cdot l_{1} \otimes l_{2}\right)$. We have:

$$
\left(s g_{1} g_{2}\right) \cdot l_{1} \otimes l_{2}=\left({ }^{s} g_{1}{ }^{s} g_{2} s\right) \cdot l_{1} \otimes l_{2}={ }^{s} g_{1} s \cdot l_{1} \otimes{ }^{s} g_{2} s \cdot l_{2}=s g_{1} \cdot l_{1} \otimes s g_{2} \cdot l_{2}=s \cdot\left(g_{1} \cdot l_{1} \otimes g_{2} \cdot l_{2}\right) .
$$

It follows that $\tilde{L} \downarrow_{H}^{G}$ and $\tilde{L}_{1} \otimes \tilde{L}_{2}$ are extensions of $X$ to $H$. So they must be isomorphic, by Theorem 3.2.

Consider $\tilde{L} \downarrow_{E}^{G}$, with $E=\left\langle w, v>\right.$ and $\mathcal{B}=\left\{Y_{i j}=\left(y_{1}^{i} \otimes 1\right) \otimes\left(y_{2}^{j} \otimes 1\right) \mid 0 \leq i, j<q\right\}$, as a $k$-basis of $\tilde{L}$. Let us write again $a, b$ and $l$ for the same integers as in the previous subsection, that is $0 \leq a, b, l<q,{ }^{w} x=x^{-1} z^{a},{ }^{w} y=y^{-1} z^{b}$ and $2 l \equiv-a(\bmod q)$. Set $v \cdot Y_{i j}=Y_{j i}$, $w \cdot Y_{i j}=\omega^{(i+j+a) b} Y_{-i-a,-j-a}$ and extend $k$-linearly this action to $\tilde{L}$. It is well defined, as we have $Y_{i j}=1 \cdot Y_{i j}=v^{2} \cdot Y_{i j}=w^{2} \cdot Y_{i j}$ and $v \cdot w \cdot Y_{i j}=\omega^{(i+j+a) b} Y_{-j-a,-i-a}=w \cdot v \cdot Y_{i j}, \forall Y_{i j} \in \mathcal{B}$. Moreover, if we define $g s \cdot Y_{i j}=g \cdot\left(s \cdot Y_{i j}\right), \forall g \in Q, \forall s \in E$ and $\forall Y_{i j} \in \mathcal{B}$, then we get a well defined $k[Q \rtimes E]$-module, as it can be easily checked that the actions of $Q$ and $E$ are compatible. So, from unicity of extensions, we deduce that we have described the (unique) action of $E$ on $\tilde{L}$.

Lemma 4.4 $\tilde{L} \downarrow_{E}^{G}$ is isomorphic to $k \oplus(k[E /<v>])^{\frac{q-1}{2}} \oplus(k[E /<v w>])^{\frac{q-1}{2}} \oplus(k E)^{\left(\frac{q-1}{2}\right)^{2}}$ as $K E$ module.

Proof. Consider the following $k$-subspaces of $\tilde{L}([\alpha]$ denotes the integer part of the real number $\alpha)$ :

$$
k<Y_{l l}>, \quad W_{i}=k<Y_{i i}, \omega^{(2 i+a) b} Y_{l-i, l-i}>, \forall\left[\frac{l+1}{2}\right] \leq i \neq l \leq\left[\frac{q+l}{2}\right],
$$




$$
\begin{gathered}
W_{i}^{\prime}=k<Y_{l+i, l-i}, Y_{l-i, l+i}>, \forall 0<i \leq \frac{q-1}{2}, \quad \text { and }, \quad \forall 0 \leq i, j<q, \text { with } \delta_{i j}=\delta_{i+j,-a}=0, \\
W_{i j}=k<Y_{i j}, Y_{j i}, \omega^{(i+j+a) b} Y_{-i-a,-j-a}, \omega^{(i+j+a) b} Y_{-j-a,-i-a}>.
\end{gathered}
$$

There are $\frac{q-1}{2}$ distinct subspaces $W_{i}$ and $W_{i}^{\prime}$, and $\left(\frac{q-1}{2}\right)^{2}$ distinct subspaces $W_{i j}$. They are all permutation $k E$-submodules of $\tilde{L}$. Indeed, $k<Y_{l l}>$ is isomorphic to the trivial $k E$-module, $W_{i}$ is isomorphic to $k<Y_{i i}>\uparrow_{<v>}^{E} \cong k\left[E /\langle v>]\right.$ as $k E$-module, because $v$ acts trivially on $W_{i}$, while $w$ acts freely, and that is true for any integer $i$ such that $\left[\frac{l+1}{2}\right] \leq i \neq l \leq\left[\frac{q+l}{2}\right]$. The same argument shows that $W_{i}^{\prime}$ is isomorphic to $k[E /<v w>]$ as $k E$-module, $\forall 0<i \leq \frac{q-1}{2}$. The subspaces $W_{i j}$ are free $k E$-modules of rank 1 , as they are isomorphic to $k<Y_{i j}>\uparrow_{1}^{E}$. Moreover, all these modules intersect trivially, as the vectors $Y_{i j}$ are linearly independent (over $k$ ) and no one appears in two different subspaces $k<Y_{l l}>, W_{i}, W_{i}^{\prime}$ or $W_{i j}$. It follows that the $k E$-submodule they generate is a direct sum and it has dimension $1+\left(\frac{q-1}{2}\right) \cdot 2+\left(\frac{q-1}{2}\right) \cdot 2+\left(\frac{q-1}{2}\right)^{2} \cdot 4=q^{2}=\operatorname{dim} \tilde{L}$. So we have $\tilde{L} \downarrow_{E}^{G} \cong k \oplus(k[E /<v>])^{\frac{q-1}{2}} \oplus(k[E /<v w>])^{\frac{q-1}{2}} \oplus(k E)^{\left(\frac{q-1}{2}\right)^{2}}$.

In particular, we deduce from this that $\tilde{L} \downarrow_{Z}^{G} \cong k \oplus(k Z)^{\left(\frac{q^{2}-1}{2}\right)}$, as $k Z$-modules, and so $S \downarrow_{Z}^{G} \cong k \oplus F$, where $Z=\langle w\rangle$ is the center of $P$ and $F$ a free $k Z$-module.

Lemma 4.5 Let $P$ be a semi-dihedral 2-group, $Z$ be its center and $M$ an indecomposable endopermutation $k P$-module with vertex $P$. Suppose that the restriction $M \downarrow_{Z}^{P}$ is an endo-trivial $k Z$ module. Then $M$ is endo-trivial.

Proof. As $M$ is indecomposable with vertex $P$, Proposition 2.1.2 in [Pu2] implies that $M$ is endotrivial, if and only if $\operatorname{Def}_{N_{P}(Q) / Q}^{N_{P}(Q)} \operatorname{Res}_{N_{P}(Q)}^{P}([M])=[k]$, for all non-trivial subgroups $Q$. If $Q$ is a non-trivial subgroup of $P$, then either $Q$ contains $Z$, or $Q$ is conjugate to $\langle v>$ and its normalizer is conjugate to $E$. In this latter case, $N_{P}(Q) / Q$ is cyclic of order 2 and $D\left(N_{P}(Q) / Q\right)=\{[k]\}$. It follows that $\operatorname{Def}_{N_{P}(Q) / Q}^{N_{P}(Q)} \operatorname{Res}_{N_{P}(Q)}^{P}([M])=[k]$.

Suppose that $Q$ contains $Z$. Then we can decompose the map $\operatorname{Def}_{N_{P}(Q) / Q}^{N_{P}(Q)} \operatorname{Res}_{N_{P}(Q)}^{P}$ as the composition $\operatorname{Def}_{N_{P}(Q) / Q}^{N_{P}(Q) / Z} \operatorname{Res}_{N_{P}(Q) / Z}^{P / Z} \operatorname{Def}_{P / Z}^{P}$. By hypothesis, $M \downarrow_{Z}^{P}$ is isomorphic to $k \oplus F$, for a free $k Z$-module $F$. Let $A=\operatorname{End}_{k} M$. Then $A$ is isomorphic to $k \oplus F^{\prime}$ as $k Z$-module, for a free $k Z$ module $F^{\prime}$. Thus we have, $\operatorname{Def}_{P / Z}^{P}([A])=\left[A^{Z} / A_{1}^{Z}\right]=[k]=\left[\operatorname{End}_{k} k\right]$, and so $\operatorname{Def}_{P / Z}^{P}([M])=[k]$. It follows that $\operatorname{Def}_{N_{P}(Q) / Q}^{N_{P}(Q) / Z} \operatorname{Res}_{N_{P}(Q) / Z}^{P / Z} \operatorname{Def}_{P / Z}^{P}([M])=[k]$ and hence $M$ is endo-trivial. Recall that $A^{Z}$ denotes the $k$-subalgebra of $A$ formed by all fixed points of $A$ under the action (by conjugacy) of $Z$, and $A_{1}^{Z}$ is the image of the relative trace map $A \longrightarrow A^{Z}, \varphi \mapsto \varphi+{ }^{w} \varphi, \forall \varphi \in A$.

Let us apply these results to the indecomposable capped endo-permutation $k P$-module $S$. Since $S \downarrow_{Z}^{P} \cong k \oplus F$, Lemma 4.5 implies that $S$ is endo-trivial. Moreover, as $S \downarrow_{E}^{P} \mid \tilde{L} \downarrow_{E}^{G}$, Lemma 4.4 implies that $S \downarrow_{E}^{P} \in[k]$.

Consider $S \downarrow_{R}^{P}$. We can write $\tilde{L} \downarrow_{R}^{G} \cong\left(\tilde{L}_{1} \otimes \tilde{L}_{2}\right) \downarrow_{R}^{H} \cong\left(\tilde{L}_{1} \downarrow_{R}^{H_{1}}\right) \otimes\left(\tilde{L}_{2} \downarrow_{R}^{H_{2}}\right)$, by transitivity of restriction and Lemma 4.3. From the previous subsection, we know that $\tilde{L}_{i \downarrow_{R}} \downarrow_{i}$ has an indecomposable direct 
summand $S_{i}$ with vertex $R$, isomorphic to $\Omega_{R}^{1}(k)$ or to $\Omega_{R}^{3}(k)$, for $i=1$ and 2 . We claim that $S_{1} \cong S_{2}$. Indeed, consider the isomorphism $\varphi$ from $H_{1}$ to $H_{2}$, mapping $h s$ to $\theta(h) \lambda(s), \forall h \in Q_{1}$ and $\forall s \in R$. Let $\operatorname{Res}_{\varphi}\left(\tilde{L}_{2}\right)$ be the $k H_{1}$-module obtained by restriction of $\tilde{L}_{2}$ via $\varphi$, i.e. where $g \cdot l$ is defined as the element $\varphi(g) \cdot l$, the latter "." denoting the action of $H_{2}$ on $\tilde{L}_{2}$, for all $g \in H_{1}$ and for all $l \in \operatorname{Res}_{\varphi}\left(\tilde{L}_{2}\right)$.

We have

$$
\operatorname{Tr}\left(z^{i}, \operatorname{Res}_{\varphi}\left(\tilde{L}_{2}\right) \downarrow_{Q^{\prime}}^{H_{1}}\right)=\operatorname{Tr}\left(\varphi\left(z^{i}\right), \tilde{L}_{2} \downarrow_{Q^{\prime}}^{H_{2}}\right)=\operatorname{Tr}\left(z^{i}, \tilde{L}_{2} \downarrow_{Q^{\prime}}^{H_{2}}\right)=q \omega^{i}=\operatorname{Tr}\left(z^{i}, \tilde{L}_{1} \downarrow_{Q^{\prime}}^{H_{1}}\right),
$$

for all $0 \leq i \leq q-1$ and so $\left(\operatorname{Res}_{\varphi}\left(\tilde{L}_{2}\right) \downarrow_{Q^{\prime}}^{H_{1}}, \tilde{L}_{1} \downarrow_{Q^{\prime}}^{H_{1}}\right)_{Q^{\prime}}=q^{2}$. Then, Lemma 3.1 and Theorem 3.2 imply that $\operatorname{Res}_{\varphi}\left(\tilde{L}_{2}\right)$ is isomorphic to $\tilde{L}_{1}$, since they both extend $L_{1}$ to $H_{1}$.

Hence, we have $\tilde{L}_{1} \downarrow_{R}^{H_{1}} \cong\left(\operatorname{Res}_{\varphi}\left(\tilde{L}_{2}\right)\right) \downarrow_{R}^{H_{1}} \cong \operatorname{Res}_{\lambda}\left(\tilde{L}_{2} \downarrow_{R}^{H_{2}}\right)$, since the restriction of $\varphi$ to $R$ is $\lambda$. Consider then the commutative diagram of $k R$-modules (with exact rows)

$$
\begin{aligned}
& 0 \longrightarrow \Omega_{R}^{1}(k) \longrightarrow k R \longrightarrow k \longrightarrow 0 \\
& 0 \longrightarrow \begin{array}{c}
\lambda \downarrow \\
\Omega_{R}^{1}(k)
\end{array} \longrightarrow \begin{array}{l}
\lambda \downarrow \\
k R
\end{array} \longrightarrow \quad \begin{array}{ll}
\operatorname{Id} \downarrow \\
k
\end{array} \quad \longrightarrow 0
\end{aligned}
$$

We deduce from this that $\operatorname{Res}_{\lambda}\left(\Omega_{R}^{1}(k)\right) \cong \Omega_{R}^{1}(k)$ and more generally that $\operatorname{Res}_{\lambda}\left(\Omega_{R}^{n}(k)\right) \cong \Omega_{R}^{n}(k)$ for all integers $n$. Therefore we have $\operatorname{Res}_{\lambda}\left(\tilde{L}_{2} \downarrow_{R}^{H_{2}}\right) \cong \tilde{L}_{2} \downarrow_{R}^{H_{2}}$. In other words, we have $\tilde{L}_{1} \downarrow_{R}^{H_{1}} \cong \tilde{L}_{2} \downarrow_{R}^{H_{2}}$ and it follows that $S_{1} \cong S_{2}$. Thus $\Omega_{R}^{2}(k) \mid S_{1} \otimes S_{2}$ and so $\Omega_{R}^{2}(k) \mid \tilde{L} \downarrow_{R}^{G}$.

Finally, $S$ is an endo-trivial $k P$-module and its restrictions to $R$ and $E$ belong to the classes of $\Omega_{R}^{2}(k)$ and $k$ respectively. By injectivity of the restriction $T(P) \longrightarrow T(R) \oplus T(E)$ (see Theorem 2.8 in [CaTh]), we conclude that $S$ is isomorphic to the indecomposable endo-trivial $k P$-module $\Omega_{P}^{1}\left(\Omega_{P /<v>}^{1}(k)\right)$, of dimension $\frac{|P|}{2}+1$.

\section{Inflation, tensor induction and tensor product}

In the previous section, we explicitly realized a few examples of endo-permutation modules as sources of simple modules for finite $p$-nilpotent groups. Now, instead of going on with explicit examples, we are going to provide tools that will allow realizations of many other modules.

\subsection{Inflation}

Let $P^{\prime}$ be a normal subgroup of $P$ and write $\bar{P}$ the quotient $P / P^{\prime}$. Let $M$ be an indecomposable endo-permutation $k \bar{P}$-module with vertex $P$, such that we can explicitly construct a finite $p$ nilpotent group $G$ having $\bar{P}$ as $p$-Sylow subgroup and a simple $k G$-module $L$ with source isomorphic to $M$. We are going to prove that we can explicitly realize $\operatorname{Inf}_{\bar{P}}^{P} M$ as source (recall that this is an indecomposable endo-permutation $k P$-module with vertex $P$, see Proposition 3.17 in [Da1]). Write $G=Q \rtimes \bar{P}$, where $Q$ is a finite $p^{\prime}$-group, and $\sigma: \bar{P} \longrightarrow \operatorname{Aut}(Q)$ the group homomorphism corresponding to the conjugation of elements of $Q$ by elements of $P$ in $G$. Consider the map $\sigma^{\prime}: P \longrightarrow \operatorname{Aut}(Q), u \mapsto \sigma(\bar{u}), \forall u \in P$. It is a group homomorphism and so defines a finite $p$-nilpotent group $G^{\prime}=Q \rtimes P$. Moreover, as $\sigma^{\prime}(u)=\operatorname{Id}_{Q}, \forall u \in P^{\prime}$, the subgroup $\{1\} \times P^{\prime}$ is 
normal in $G^{\prime}$ and the quotient is isomorphic to $G$. Let $L^{\prime}=\operatorname{Inf}_{G}^{G^{\prime}} L$. It is a simple $k G^{\prime}$-module with vertex $P$ and its source is isomorphic to $\operatorname{Inf}_{\bar{P}}^{P} M$. Indeed, we have by hypothesis $M \mid L \downarrow_{\bar{P}}^{G}$, and so $\operatorname{Inf}_{\bar{P}}^{P} M \mid \operatorname{Inf}_{\bar{P}}^{P}\left(L \downarrow_{\bar{P}}^{G}\right) \cong L^{\prime} \downarrow_{P}^{G^{\prime}}$.

\subsection{Tensor induction}

Let $C$ be a subgroup of $P$ and $M$ an indecomposable endo-permutation $k C$-module with vertex $C$, such that we can explicitly construct a finite $p$-nilpotent group $G$ with a $p$-Sylow subgroup isomorphic to $C$, and a simple $k G$-module $L$ with source isomorphic to $M$. Write $G=Q \rtimes C$, where $Q$ has order prime to $p$. Suppose moreover that the restriction $L \downarrow_{Q}^{G}$ is a simple $k Q$-module. In this section, we are going to realize the cap of $\operatorname{Ten}_{C}^{P} M$ as source of a simple module for a finite $p$-nilpotent group having $P$ as $p$-Sylow subgroup. The constructions we are going to do involve a choice of a set of representatives of the left cosets of $P / C$. We can avoid this if we take the functorial point of view of tensor induction, introduced by S. Bouc (see Remark 5.1 below). However, since we didn't introduce this formalism before, we prefer to write here a direct proof.

Let $[P / C]=\left\{v_{0}, \ldots, v_{r}\right\}$ be a set of representatives of the left cosets of $P / C$ and assume $v_{0}=1$. For each $0 \leq i \leq r$, we formally define a group ${ }^{v_{i}} Q=\left\{{ }^{v_{i}} g \mid g \in Q\right\}$, where the composition law is given by ${ }^{v_{i}} g{ }^{v_{i}} h=v(g h), \forall g, h \in Q$. Let $H=\prod_{i=0}^{r}{ }^{v_{i}} Q$. We let $P$ act on $H$ as follows. Let $\left({ }^{v_{i}} h_{i}\right)_{i=0}^{r} \in H$ and $u \in P$. As left multiplication by $u$ acts by permutation on $[P / C]$, there exists an unique $\sigma_{u} \in S_{r+1}$ (where $S_{r+1}$ acts on $\{0, \ldots, r\}$ ), such that $u v_{i}=v_{\sigma_{u}(i)} c(u, i)$ for a unique $c(u, i) \in C, \forall 0 \leq i \leq r$. We set then ${ }^{u}\left(v^{v_{i}} h_{i}\right)_{i=0}^{r}=\left({ }^{v_{i}}\left(c\left(u, \sigma_{u}^{-1}(i)\right) h_{\sigma_{u}^{-1}(i)}\right)\right)_{i=0}^{r}$. We can then check directly that ${ }^{1} h=h$ and that ${ }^{\left(u u^{\prime}\right)} h=u^{u}\left(u^{\prime} h\right), \forall h \in H, \forall u, u^{\prime} \in P$ (as $\sigma_{u u^{\prime}}=\sigma_{u} \sigma_{u^{\prime}}$ and $\left.c\left(u u^{\prime}, i\right)=c\left(u, \sigma_{u^{\prime}}(i)\right) c\left(u^{\prime}, i\right), \forall 0 \leq i \leq r\right)$. So we have a $p$-nilpotent group $G^{\prime}=H \rtimes P$, in which the groups ${ }^{v_{i}} Q, \forall 0 \leq i \leq r$ are all conjugate. Moreover, for all $0 \leq i \leq r$, the $k\left[{ }^{v_{i}} Q\right]$-module $v_{i}\left(L \downarrow_{Q}^{G}\right)$ is simple and so, as $k$ is algebraically closed, the external tensor product $\tilde{L}=\bigotimes_{i=0}^{r} v_{i}\left(L \downarrow_{Q}^{G}\right)$ is a simple $k H$-module.

Set, for all $u \in P$ and for all $x=\bigotimes_{i=0}^{r} x_{i} \in \tilde{L}, u * x=\bigotimes_{i=0}^{r} v_{i}\left(c\left(u, \sigma_{u}^{-1}(i)\right) \cdot x_{\sigma_{u}^{-1}(i)}\right)$, where - denotes the action of $G$ on $L$. Then, $\tilde{L}$ turns out to be a $k P$-module if we extend this action $k$-linearly to $\tilde{L}$. Indeed, $\forall u, u^{\prime} \in P$ and $\forall x=\otimes_{i=0}^{r} x_{i} \in \tilde{L}$, we have $1 * x=x$ et $u *\left(u^{\prime} * x\right)=$

$$
\begin{gathered}
=u *\left(\bigotimes_{i=0}^{r} v_{i}\left(c\left(u^{\prime}, \sigma_{u^{\prime}}^{-1}(i)\right) \cdot x_{\sigma_{u^{\prime}}^{-1}(i)}\right)\right)=\bigotimes_{i=0}^{r} v_{i}\left(c\left(u, \sigma_{u}^{-1}(i)\right) \cdot\left(c\left(u^{\prime}, \sigma_{u^{\prime}}^{-1}\left(\sigma_{u}^{-1}(i)\right)\right) \cdot x_{\sigma_{u^{\prime}}^{-1}\left(\sigma_{u}^{-1}(i)\right)}\right)\right)= \\
=\bigotimes_{i=0}^{r} v_{i}\left(\left(c\left(u, \sigma_{u}^{-1}(i)\right) c\left(u^{\prime}, \sigma_{u^{\prime}}^{-1}\left(\sigma_{u}^{-1}(i)\right)\right)\right) \cdot x_{\sigma_{u^{\prime}}^{-1}\left(\sigma_{u}^{-1}(i)\right)}\right)=\bigotimes_{i=0}^{r} v_{i}\left(c\left(u u^{\prime}, \sigma_{u u^{\prime}}^{-1}(i)\right) \cdot x_{\sigma_{u u^{\prime}}(i)}\right)=
\end{gathered}
$$

$=\left(u u^{\prime}\right) * x$.

Then, if we set $(h u) * x=h \cdot(u * x), \forall h \in H, \forall u \in P$ and $\forall x \in \tilde{L}$, the actions of $P$ and $H$ are compatible, and so $\tilde{L}$ becomes a $k G^{\prime}$-module. Indeed, $\forall h \in H, \forall u \in P$ and $\forall x \in \tilde{L}$, we have:

$(u h) * x=\bigotimes_{i=0}^{r} v_{i}\left(c\left(u, \sigma_{u}^{-1}(i) h_{\sigma_{u}^{-1}(i)}\right) \cdot(u * x)=\bigotimes_{i=0}^{r} v_{i}\left(c\left(u, \sigma_{u}^{-1}(i)\right) h_{\sigma_{u}^{-1}(i)} \cdot\left(c\left(u, \sigma_{u}^{-1}(i)\right) \cdot x_{\sigma_{u}^{-1}(i)}\right)\right)=\right.$ 


$$
=\bigotimes_{i=0}^{r} v_{i}\left(c\left(u, \sigma_{u}^{-1}(i)\right) h_{\sigma_{u}^{-1}(i)} \cdot x_{\sigma_{u}^{-1}(i)}\right)=u *(h \cdot x) .
$$

Moreover, we have $\tilde{L} \downarrow_{P}^{G^{\prime}} \cong \operatorname{Ten}_{C}^{P}\left(L \downarrow_{C}^{G}\right)$, as they are isomorphic as $k$-vector spaces (of dimension $(\operatorname{dim} L)^{r+1}$ ) and as the action of $P$ on the generators coincides on both modules. As the $k\left[{ }^{v_{i}} C\right]$ module ${ }^{v_{i}} M$ is a direct summand of $v_{i}\left(L \downarrow_{C}^{G}\right)$, for all $0 \leq i \leq r$, it follows that $\operatorname{Ten}_{C}^{P} M \mid \operatorname{Ten}_{C}^{P}\left(L \downarrow_{C}^{G}\right)$ as $k P$-modules and so the source of $\tilde{L}$ is isomorphic to the cap of $\operatorname{Ten}_{C}^{P} M$.

Remark 5.1 If we consider the functorial point of view of tensor induction introduced by S. Bouc in [Bo], then we do not need to choose a set of representatives of the left cosets of $P / C$. Indeed, the group $H$ we defined above is isomorphic to the group $\operatorname{Hom}_{C}(P, Q)$ of $C$-equivariant maps from $P$ to $Q$, where we consider $P$ and $Q$ as left $C$-sets for the left multiplication inside $P$ and respectively for the action of $C$ on $Q$ (i.e. conjugation inside $G$ ).

Similarly, the $k H$-module $\tilde{L}$ is isomorphic to a quotient of $k \operatorname{Hom}_{C}(P,\{L\})$, where $\{L\}$ denotes the underlying $C$-set of $L$ (in the notations of [Bo], we have $\tilde{L} \cong t_{P}(L)$ ). Then, applying Lemma 9.9 and Remark 9.10 of [Bo], we get the same result we proved above (namely, that $\tilde{L}$ is a simple $k G^{\prime}$ module and its source is isomorphic to the cap of $\operatorname{Ten}_{C}^{P} L$ ) and this proof does not involve any choice of representatives.

Remark 5.2 Let $G=Q \rtimes C$ be a finite $p$-nilpotent group, with $Q$ having order prime to $p$, and $C$ a p-group, and let $L$ be a simple $k G$-module. We can drop the additional hypothesis we made above, namely that $L \downarrow_{Q}^{G}$ is a simple $k Q$-module, if we modify the construction of $\tilde{L}$ as follows. If $S$ is a simple $k Q$-module belonging to a block e of $k Q$ and $I=\left\{g \in G \mid{ }^{g} S \cong S\right\}$ is the inertial subgroup of $S$, then, by Clifford theory, we know that $S$ can be extended to a simple kI-module $\tilde{S}$ (which belongs to the block e of $k I$ ). Moreover, the relative trace from $I$ to $G$ sends e on a block $b$ of $k G$ and the $k$-algebras $k I e$ and $k G b$ are Morita equivalent. This equivalence lets $\tilde{S}$ correspond to the simple $k G$-module $\operatorname{Ind}_{I}^{G}(\tilde{S})$. It follows that, if $L$ is a simple $k G$-module, then its restriction to $k Q$ is not a simple module, in general. However, $L_{Q}{ }_{Q}^{G}$ is a finite direct sum $\oplus_{i=0}^{r}{ }^{v_{i} S}$ of conjugate simple modules (we can assume that $v_{0}=1$ ), having conjugate inertial subgroups $I_{i}$, which are strictly contained in $G$. We also know that $L \cong \operatorname{Ind}_{I_{0}}^{G} \tilde{S}$ and so, in this situation, instead of constructing the simple $k G^{\prime}$-module $\tilde{L}$ using $L \downarrow_{Q}^{G}$, we consider the simple $k Q$-module $S$. Notice, however, that in every situation we realized until now, the additional hypothesis we make here was fulfilled.

\subsection{Tensor product}

Let $\mathcal{C}$ be a family of indexes (e.g. $\mathcal{C}$ is a set of conjugacy classes of non-trivial cyclic subgroups of $P)$. Suppose that for each $C \in \mathcal{C}$ there is an indecomposable endo-permutation $k P$-module $M_{C}$ with vertex $P$, such that we can explicitly construct a $p$-nilpotent group $G_{C}=Q_{C} \rtimes P\left(Q_{C}\right.$ of order prime to $p$ ) and a simple $k G_{C}$-module $L_{C}$ with source isomorphic to $M_{C}$. We prove the following result.

Theorem 5.3 If $L_{C} \downarrow_{Q_{C}}^{G_{C}}$ is a simple $k Q_{C}$-module, $\forall C \in \mathcal{C}$, then there exists a p-nilpotent group $G$ and a simple $k G$-module $\tilde{L}$ with source isomorphic to the cap of $\otimes_{C \in \mathcal{C}} M_{C}$. 
Proof. Let $G=\left(\prod_{C \in \mathcal{C}} Q_{C}\right) \rtimes P$, where $P$ acts diagonally on $\prod_{C \in \mathcal{C}} Q_{C}$, i.e.

$$
{ }^{u}\left(h_{C}\right)_{C \in \mathcal{C}}=\left({ }^{u} h_{C}\right)_{C \in \mathcal{C}}, \forall u \in P, \forall\left(h_{C}\right)_{C \in \mathcal{C}} \in \prod_{C \in \mathcal{C}} Q_{C} .
$$

This is a well defined semi-direct product, as $P \cap\left(\prod_{C \in \mathcal{C}} Q_{C}\right)=\{1\},{ }^{1}\left(h_{C}\right)_{C \in \mathcal{C}}=\left(h_{C}\right)_{C \in \mathcal{C}}$ and ${ }^{\left(u u^{\prime}\right)}\left(h_{C}\right)_{C \in \mathcal{C}}=\left({ }^{\left(u u^{\prime}\right)} h_{C}\right)_{C \in \mathcal{C}}=\left({ }^{u}\left(u^{\prime} h_{C}\right)\right)_{C \in \mathcal{C}}={ }^{u}\left(u^{\prime}\left(h_{C}\right)_{C \in \mathcal{C}}\right), \forall u, u^{\prime} \in P$ and $\forall\left(h_{C}\right)_{C \in \mathcal{C}} \in$ $\prod_{C \in \mathcal{C}} Q_{C}$. The external tensor product (over $k$ ) $L=\bigotimes_{C \in \mathcal{C}}\left(L_{C} \downarrow_{Q_{C}}^{G_{C}}\right)$ is a simple $k\left[\prod_{C \in \mathcal{C}} Q_{C}\right]-$ module, as $k$ is algebraically closed. Moreover, we can extend $L$ to a simple $k G$-module $\tilde{L}$, by letting $P$ act diagonally: $u \cdot \otimes_{C \in \mathcal{C}} l_{C}=\otimes_{C \in \mathcal{C}} u \cdot l_{C}$, and setting then $\left(\left(h_{C}\right)_{C \in \mathcal{C}} u\right) \cdot \otimes_{C \in \mathcal{C}} l_{C}=$ $\left(h_{C}\right)_{C \in \mathcal{C}} \cdot\left(u \cdot \otimes_{C \in \mathcal{C}} l_{C}\right), \forall u \in P, \forall\left(h_{C}\right)_{C \in \mathcal{C}} \in \prod_{C \in \mathcal{C}} Q_{C}$ and $\forall \otimes_{C \in \mathcal{C}} l_{C} \in \tilde{L}$, where $\cdot$ denotes the action of $P$ on the $k G_{C}$-module $L_{C}, \forall C \in \mathcal{C}$. Next calculation shows that the actions of $P$ and $\prod_{C \in \mathcal{C}} Q_{C}$ are compatible:

$\left(u\left(h_{C}\right)_{C \in \mathcal{C}}\right) \cdot \otimes_{C \in \mathcal{C}} l_{C}=\left({ }^{u} h_{C}\right) \cdot u \cdot \otimes_{C \in \mathcal{C}} l_{C}=\otimes_{C \in \mathcal{C}} u \cdot h_{C} \cdot l_{C}=u \cdot \otimes_{C \in \mathcal{C}} h_{C} \cdot l_{C}=u \cdot\left(h_{C}\right)_{C \in \mathcal{C}} \cdot \otimes_{C \in \mathcal{C}} l_{C}$.

So we get a finite $p$-nilpotent group $G$, with $P$ as $p$-Sylow subgroup and a simple $k G$-module $\tilde{L}$, with vertex $P$, because the dimension of each $L_{C}$, and thus the dimension of $\tilde{L}$, is prime to $p$. By hypothesis, $M_{C}$ is a direct summand of $L_{C} \downarrow_{P}^{G_{C}}, \forall C \in \mathcal{C}$. It follows that $\bigotimes_{C \in \mathcal{C}} M_{C}$ is a direct summand of $\bigotimes_{C \in \mathcal{C}}\left(L_{C} \downarrow_{P}^{G_{C}}\right)$, which is clearly isomorphic to $\tilde{L} \downarrow_{P}^{G}$ as $k P$-module. Hence, the source of the simple $k G$-module $\tilde{L}$ is isomorphic to the cap of $\otimes_{C \in \mathcal{C}} M_{C}$.

Let us apply this to the following two examples.

Corollary 5.4 Let $p=2$ and $P$ be a quaternion 2-group. Then every indecomposable torsion endopermutation $k P$-module with vertex $P$ can be explicitly realized as source of a simple $k G$-module for a finite 2-nilpotent group $G$ having $P$ as 2-Sylow subgroup.

Proof. It follows by [CaTh] that every indecomposable torsion endo-permutation module belongs to the class of an endo-trivial module. More precisely, we have $T^{t}(P) \cong \mathbb{Z} / 4 \mathbb{Z} \times \mathbb{Z} / 2 \mathbb{Z}$, where $\mathbb{Z} / 4 \mathbb{Z}$ is generated by the class of a module of dimension $|P|-1$ and $\mathbb{Z} / 2 \mathbb{Z}$ by the class of an "exceptional" endo-trivial module, of dimension $\frac{|P|}{2}+1$. Thus, as we noticed in section 4 , the classes of the two modules we realized in section 3 generate the whole torsion subgroup $D^{t}(P)$ of $D(P)$ and so, by Theorem 5.3, every indecomposable torsion endo-permutation $k P$-module with vertex $P$ can be explicitly realized (up to isomorphism).

Corollary 5.5 Let $p$ be an odd prime and $P$ a metacyclic p-group. Then every indecomposable torsion endo-permutation $k P$-module with vertex $P$ can be explicitly realized as source of a simple $k G$-module for a finite p-nilpotent group $G$ having $P$ as p-Sylow subgroup.

Proof. By the main theorem of $[\mathrm{Ma}]$, the set $\left\{\left[\operatorname{Ten}_{C}^{P} \operatorname{Inf}_{C / \Phi(C)}^{C} \Omega_{C / \Phi(C)}^{1}(k)\right], C \in \mathcal{C}\right\}$, where $\mathcal{C}$ is the set of conjugacy classes of non-trivial cyclic subgroups of $P$, forms a basis of the $\mathbb{F}_{2}$-vector space $D^{t}(P)$ (all torsion elements have order 2). In other words, every indecomposable torsion 
endo-permutation $k P$-module with vertex $P$ is isomorphic to a direct summand of a $k P$-module of the form $\otimes_{C \in \mathcal{C}^{\prime}} \operatorname{Teninf}_{C / \Phi(C)}^{P} \Omega_{C / \Phi(C)}^{1}(k)$, where $\mathcal{C}^{\prime} \subseteq \mathcal{C}$. In section 3 , we realized explicitly $\Omega_{C / \Phi(C)}^{1}(k)$ as source, as $C / \Phi(C)$ is cyclic of order $p$. Thus, by subsections 5.1 and 5.2 , we can do the same for the cap of each $\operatorname{Teninf}_{C / \Phi(C)}^{P} \Omega_{C / \Phi(C)}^{1}(k)$, where $C \in \mathcal{C}$, and this construction satisfies the hypothesis of Theorem 5.3. It follows that every indecomposable torsion endo-permutation $k P$-module with vertex $P$ can be explicitly realized as source.

\section{Conclusion}

If we sum up the results we obtained in the previous sections, then we come to the following conclusion.

Theorem 6.1 Let $p$ be a prime, $P$ a finite $p$-group and $k$ an algebraically closed field of characteristic $p$. Then the cap of any $k P$-module of the form $\bigotimes_{(Q / R) \in \mathcal{S}} \operatorname{Ten}_{Q}^{P} \operatorname{Inf}_{Q / R}^{Q}\left(M_{Q / R}\right)$, where $M_{Q / R}$ is an indecomposable torsion endo-trivial module with vertex $Q / R$, and $\mathcal{S}$ is a set of sections of $P$ that are cyclic, or also quaternion or semi-dihedral, in case $p=2$, can be explicitly realized as source of a simple module with vertex $P$ for some finite p-nilpotent group.

Proof. By section 5, if we know how to realize explicitly $M_{Q / R}, \forall Q / R \in \mathcal{S}$, then we are able to realize the cap of a tensor product $\bigotimes_{Q / R \in \mathcal{S}} \operatorname{Ten}_{Q}^{P} \operatorname{Inf}_{Q / R}^{Q}\left(M_{Q / R}\right)$. Thus, it is enough to check that we have an explicit realization for any indecomposable torsion endo-trivial module with vertex $P$, for a finite cyclic, quaternion or semi-dihedral $p$-group $P$. And, in fact, we just need to consider a set of modules whose classes generate the torsion subgroup $T^{t}(P)$ of $T(P)$, as tensor product corresponds to linear combination in $D(P)$. If $P$ is cyclic, then $T^{t}(P)$ is generated by the class of $\Omega_{P}^{1}(k)$, that we realized as our first example. If $p=2$ and $P$ is quaternion, then the statement is proved in Corollary 5.4. Finally, if $p=2$ and $P$ is semi-dihedral, then, $T^{t}(P)$ is cyclic of order 2, generated by the class of the module we realized explicitly in the semi-dihedral example. So the statement is proved.

Let us now take into account Theorem 8.39 and Remark 8.41 in [Pu1]. We can sum up them into the following statement: let $p$ be a prime, $k$ an algebraically closed field with characteristic $p$ and $G$ a finite $p$-solvable group having a $p$-Sylow subgroup $P$ and a simple $k G$-module $L$ with vertex $P$. Then, the source of $L$ is the cap of a module of the shape $\bigotimes_{Q / R \in \mathcal{S}} \operatorname{Ten}_{Q}^{P} \operatorname{Inf}_{Q / R}^{Q}\left(M_{Q / R}\right)$, where the set $\mathcal{S}$ and the modules $M_{Q / R}$ are as in Theorem 6.1. Hence, the following corollary is straightforward.

Corollary 6.2 Let $p$ be a prime, $P$ a finite p-group and $k$ an algebraically closed field of characteristic $p$. Then any indecomposable endo-permutation $k P$-module $M$ with vertex $P$ that can be a source of a simple module with vertex $P$ for a finite $p$-solvable group can be explicitly realized as source for a finite p-nilpotent group. 
Finally, we would like to come back to the last remark of the introduction to draw the main consequence of our theorem, in case both following conjectures hold, namely that, if the source of a simple module is an endo-permutation module, then its class should be a torsion element in the Dade group, and that there are no other indecomposable torsion endo-permutation modules as the ones that are isomorphic to the cap of a module of the form $\bigotimes_{Q / R \in \mathcal{S}} \operatorname{Ten}_{Q}^{P} \operatorname{Inf}_{Q / R}^{Q}\left(M_{Q / R}\right)$, as in our main theorem. Following Jon Carlson's recent result, this latter conjecture holds for $p$ odd and so, if the first conjecture is also true, then we would have proved that any torsion endo-permutation module is the source of a simple module and it can be explicitly realized as it for a finite $p$-nilpotent group $(p$ odd).

\section{References}

[Bo] S. Bouc, Non-Additive Exact Functors and Tensor Induction for Mackey Functors, AMS Memoirs 683, vol. 144 (2000), 275-349.

[BoTh] S. Bouc, J. Thévenaz, The group of endo-permutation modules, Invent. math. 139 (2000), 275-349.

[CaTh] J. Carlson, J. Thévenaz, Torsion endo-trivial modules, Algebr. Represent. Theory 3 (2000), 303-335.

[CR] C. W. Curtis, I. Reiner, Methods of representation theory with applications to finite groups and orders I, Pure and applied mathematics, John Wiley and Sons, 1981.

[Da1] E. C. Dade, Endo-permutation modules over p-groups, I, II, Ann. Math. 107 (1978), 459494, 108 (1978), 317-346.

[Da2] E. C. Dade, Blocks with cyclic defect groups I, Ann. Math. 84 (1966), 20-48.

[DH] K. Doerk, T. Hawkes, Finite solvable groups, Walter de Gruyter, 1992.

[Fe] W. Feit, The Representation Theory of Finite groups, North Holland Publishing Company, 1982.

[Go] D. Gorenstein, Finite groups, Harper and Row, 1968.

[Hu] B. Huppert, Character theory of finite groups, Walter de Gruyter, 1998.

[Is] I. M. Isaacs, Character theory of finite groups, Acad. Press, 1976.

[Ma] N. Mazza, The Dade group of a metacyclic p-group, J. Algebra, to appear.

[Pu1] L. Puig, Notes sur les p-algèbres de Dade, Preprint, 1988.

[Pu2] L. Puig, Affirmative answer to a question of Feit, J. Algebra 131 (1990), 513-526.

[Se] J.-P. Serre, Représentation linéaires des groupes finis, Hermann, 1978.

[Th] J. Thévenaz, G-algebras and modular representation theory, Oxford, 1995. 